\title{
Multidimensional signal detection analyses (MSDA) for testing separability and independence: A Pascal program
}

\author{
HELENA KADLEC \\ University of Victoria, Victoria, British Columbia, Canada
}

\begin{abstract}
A Pascal (Turbo 6.0) program for testing perceptual separability, decisional separability, and perceptual independence - three types of independence defined in the context of general recognition theory (GRT) - is presented. GRT is essentially a multidimensional extension of signal detectability theory (Green \& Swets, 1966) and has been employed in complete-identification experimental paradigms to examine whether information processing of stimulus dimensions is perceptually and/or decisionally separable and/or perceptually independent. The definitions of these different forms of "independence" are provided, the experimental design is described, and the program, MSDA, is described and demonstrated.
\end{abstract}

Complete-identification experiments are conveniently employed in studies of perception to investigate whether or not various stimulus attributes, or dimensions, are processed "independently" of each other. In these experiments, a minimum of two dimensions of interest are specified and a minimum of two levels are selected for each dimension. For example, in visual perception, one may be interested in studying whether the two dimensions of shape and hue are processed independently. Selecting two levels for each dimension requires two shapes (e.g., a rectangle and an ellipse) and two levels of hue (e.g., orange and red). The stimulus set is then constructed by factorially combining all levels of each dimension with all levels of the second; we have been calling this the feature-complete factorial design (Kadlec \& Townsend, 1992a, 1992b). In the present example, the stimulus set would consist of an orange rectangle, an orange ellipse, a red rectangle, and a red ellipse. This is akin to stimulus sets employed by, for example, Garner and his colleagues to study perceptual separability in speeded classification experiments (e.g., Garner, 1974).

In complete-identification experiments, each stimulus of the set has a unique response, and upon a presentation of the stimulus, the observer must identify all aspects of the stimulus. Thus, for each stimulus, the correct response, along with each type of possible error, is summarized into a confusion matrix (e.g., Townsend \& Landon, 1983). The confusion matrix provides the raw data that are used to infer support for or failure of independent processing of the stimulus dimensions under study. (Table 3 shows an example of a confusion matrix, which is discussed further below.)

This work was partially supported by Grant OPG0121280 from NSERC of Canada. The author gratefully acknowledges the helpful suggestions of Roger Graves and William Lee on an earlier draft of this paper. Correspondence should be addressed to $\mathrm{H}$. Kadlec, Department of Psychology, P. O. Box 3050, University of Victoria, Victoria, BC, Canada V8W 3P5 (e-mail: hkadlec@uvaix.uvic.ca).
Two issues arise in this context. The first pertains to the definitions of independence of processing, and the second concerns inferences, from the data, about whether the two (or more) dimensions are processed independently. One tack (e.g., Garner, 1974; Garner \& Felfoldy, 1970; Pomerantz \& Pristach, 1988) is to define two dimensions as "perceptually separable," a type of independence, if (1) one of the tested dimensions has no interfering effects on the other dimension(s) when subjects are asked to classify the stimuli by only one dimension while ignoring variations of the second (filtering task), and (2) there are no facilitatory effects of one dimension on the other, or redundancy gains, in tasks where the two dimensions are correlated (i.e., have the same level). This operational definition of independent processing (i.e., perceptual separability of Garner), however, while useful, is theoretically unsatisfying, and clearer definitions of independence are now available in the context of general recognition theory (GRT; e.g., Ashby \& Townsend, 1986).

It is the purpose of this paper to briefly present three definitions of independence of GRT and their relationships to some testable conditions and to offer a computer program that estimates and tests the observable conditions that allow inferences to be drawn about these three types of independence. The definitions and the relationships have been reported elsewhere (see Ashby \& Townsend, 1986; Kadlec \& Townsend, 1992a); they are briefly recapitulated here for the reader's reference. Readers familiar with GRT can skip the next section without loss of continuity. The computer program is the primary and novel contribution in this paper.

\section{DEFINITIONS OF INDEPENDENCE AND SIGNAL DETECTION PARAMETERS}

Three theoretical definitions of independence of perceptual dimensions have been proposed within GRT (Ashby \& Townsend, 1986): perceptual separability, decisional separability, and perceptual independence. These 
notions have theoretical links to a number of observable conditions, particularly, marginal response invariance (MRI) and sampling independence (SI). Furthermore, because GRT is essentially a multidimensional extension of signal detection theory (Green \& Swets, 1966), the sensitivity $d^{\prime}$ and response bias $\beta$ parameters of SDT provide further tests of these types of independence (Kadlec \& Townsend, $1992 \mathrm{a}, 1992 \mathrm{~b}$ ). The following is a brief description of GRT and the different types of independence and their relationships to the observable conditions and two types of signal detection parameters.

\section{Notation and Assumptions of GRT}

In general, we denote $m$ stimulus dimensions as $A, B$, $\ldots, M$, and the levels on each dimension are given by subscripts. For example, the set of stimuli for the completeidentification experiment, constructed from dimensions $A$ and $B$ (each with two levels), contains four stimuli: $A_{1} B_{1}$, $A_{1} B_{2}, A_{2} B_{1}$, and $A_{2} B_{2}$. As in Thurstone's law of comparative judgment (Thurstone, 1927) or in signal detection theory (Green \& Swets, 1966), it is assumed that each stimulus is perceived slightly differently each time it is presented to the observer, and thus a distribution of perceptual effects (perhaps due to internal noise) results for each. Under the additional assumption that each stimulus dimension has a perceptual dimension associated with it, which is a common assumption made in these types of studies, we represent the distribution of the perceptual effects for each multidimensional stimulus as a multivariate probability density, $f_{i j}$. In general, no assumptions are made about the particular forms of these densities. Here, however, because Gaussian signal detection estimates are employed, the distributions are assumed to be Gaussian.

An example of a perceptual space for four stimuli, each having one of two possible levels on two dimensions and combined factorially, is shown in Figure 1. The top panel of Figure 1 shows the four bivariate Gaussian densities, one for each stimulus. The bottom panel of Figure 1 shows a more convenient representation of the perceptual space, in terms of equal density contours; these are obtained by slicing through the three-dimensional densities, as if looking at these from above. Any point inside the elliptical contours has a higher probability of occurrence, whereas points outside the contours have a lower probability of occurrence. The shapes of these contours provide information regarding the relative sizes of the variances along the two dimensions and about the correlation: Circular contours indicate equal variances and a zero correlation; vertical (or horizontal) ellipses correspond to unequal variances with larger variance along the $y$ - $(x$-) axis; and tilted ellipses indicate that there is a nonzero correlation between the dimensions.

On a given trial, the stimulus $\left(A_{i} B_{j} \ldots\right)$ gives rise to a percept that is a point $(x, y, \ldots)$ in the perceptual space. As in signal detection theory, the observer has a criterion, $c_{M}$, for each dimension $M$ such that by comparing the percept to each criterion, a decision regarding the identification of the stimulus is made. For example, if there are two levels on each of two dimensions, $A$ and $B$, and thus four stim-
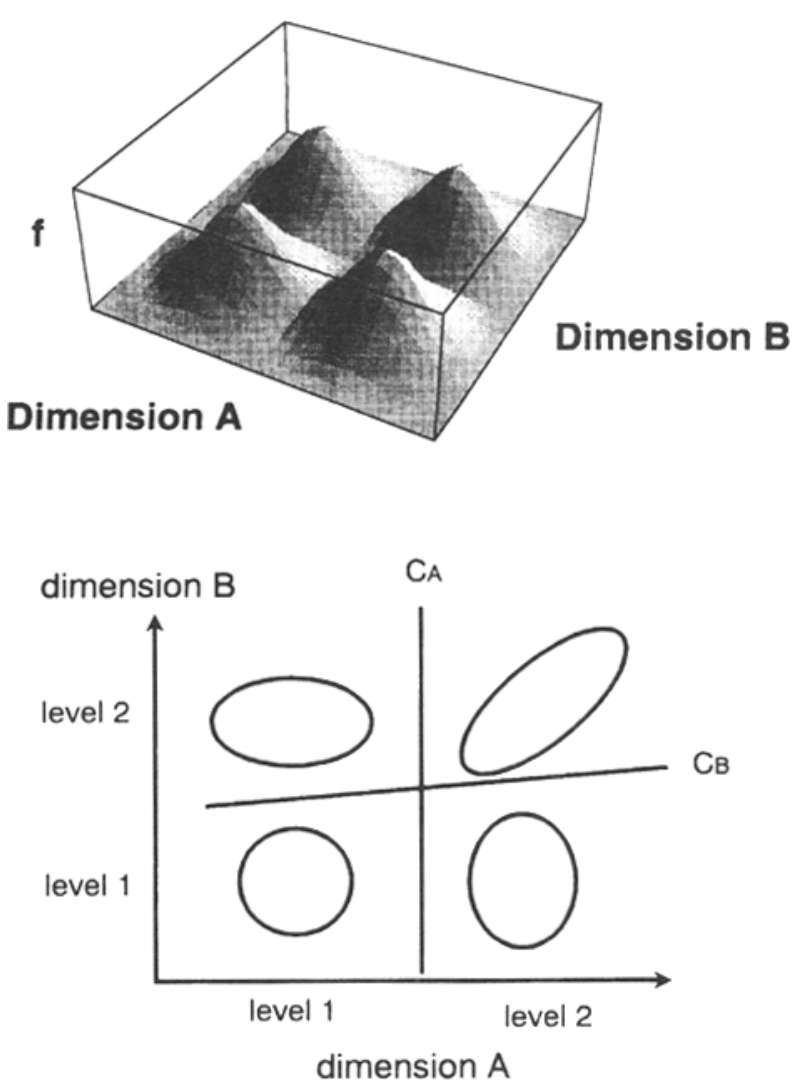

Figure 1. Example of a representation of four stimuli in twodimensional perceptual space. The top panel shows the bivariate distributions of perceptual effects for each of the four stimuli; the bottom panel shows equal density contours of the distributions.

uli, four response regions are created. If the percept falls above the criterion $c_{A}$ for dimension $A$ and below the criterion $c_{B}$ for dimension $B$, the observer responds that he/she saw stimulus $A_{2} B_{1}$.

\section{Definitions}

The term perceptual independence (PI) is defined as the stochastic independence of two (or more) random variables that represent the perceptual dimensions within a given stimulus - that is, when the joint perceptual effect is equal to the product of the marginal effects. For two dimensions, $\mathrm{PI}$ in stimulus $A_{i} B_{j}$ is defined as

$$
f_{i j}(x, y)=g_{i j}(x) \cdot g_{i j}(y), \text { for all } x, y,
$$

where $g_{i j}(x)$ and $g_{i j}(y)$ are marginal densities for dimensions $A$ and $B$, respectively, in stimulus $A_{i} B_{j}$. For more dimensions, PI would simply be defined to hold if the joint perceptual effect was equal to the product of all marginal effects.

PI is a within-stimulus type of independence of two (or more) dimensions and is related to the specific levels on the dimensions. For example, "red" may be perceptually independent of "rectangle" when the observer is presented with a stimulus that is a red rectangle; however, "orange" may not be perceptually independent of "rectangle" in a 
stimulus that is an orange rectangle. Thus, the dimensions of color and shape in this example are perceptually independent but only at specific levels of color and shape (red and rectangle).

The second type of PI, perceptual separability (PS), is an across-stimulus independence. Formally, it is defined in terms of the marginal densities, $g_{i j}$; if the marginal densities for each level of one dimension are equal across all levels of the other dimension(s), that dimension is said to be perceptually separable from the other dimension(s):

$$
g_{i 1}(x)=g_{i 2}(x), \text { for } i=1 \text { and } 2 \text {. }
$$

PS refers to independence of dimensions in the entire stimulus set. For example, if the perception of color (red and orange) produces the same distributions of perceptual effects regardless of what the shapes of the stimuli were (rectangle or ellipse), then color is said to be perceptually separable from shape.

Furthermore, note that PI (Equation 1) is a symmetric relation between two dimensions: if "red" is perceptually independent of "rectangle," then "rectangle" is also perceptually independent of "red." PS (Equation 2), however, is not necessarily symmetrical; perception of color can be perceptually separable from shape, but shape need not be perceptually separable from color.

Because behavioral measures of perception involve both perceptual and decisional processes, a notion of decisional independence is also considered (Ashby \& Townsend, 1986). Decisional separability (DS) occurs when the subject's decision about one dimension is unaffected by the levels of the other dimension(s). In the GRT representation, this corresponds to decision criteria that are parallel to the perceptual axes. This means that the decision about, for example, the color of the stimulus will not depend where on the shape continuum the percept happened to fall. As was the case for PS, DS is not necessarily a symmetric relation for perceptual dimensions; one dimension may be decisionally separable from the other, but not vice versa.

When three or more dimensions compose a given stimulus set, PS and DS can be defined in two alternative ways (Kadlec \& Townsend, 1992b). Two dimensions $(A$ and $B$ ) may be jointly (pairwise) perceptually separable from a third $(C)$ when the joint perceptual distributions for the two dimensions are identical regardless of the level of the third dimension. In other words, the third dimension $(C)$ has no effect on the joint perception of the $A_{i} B_{j}$ combination.

Alternatively, one dimension may exhibit single-component $P S$ when its perception is unaffected by changes in all other dimensions. This single-component analysis allows a direct look at how each individual dimension is influenced by changes in levels of all other dimensions in the stimulus set.

PI, PS, and DS are theoretical definitions of independence, and the underlying joint densities are not observable. However, theoretical relationships between these and a number of observable measures have been shown. In a series of theorems, Ashby and Townsend (1986) have linked these concepts of independence with a number of behaviorally defined notions of independence. Two are relevant for accuracy data and are based on probabilities derived from a confusion matrix: marginal response invariance and sampling independence. A second set of derived measures consists of two types of signal detection parameters - marginal and conditional- that have been shown to be tied to PS, DS, and PI (Kadlec \& Townsend, 1992a).

Marginal response invariance (MRI) is defined as occurring when the probability of correctly reporting one component in a given stimulus does not depend on the level of the other component(s) in the stimulus - that is, MRI holds whenever

$$
P\left(a_{i} \mid A_{i} B_{j}\right)=P\left(a_{i} \mid A_{i} B_{k}\right), i=1,2, \ldots ; j \neq k,
$$

where $P\left(a_{i}\right)=P\left(a_{i} b_{1}\right)+P\left(a_{i} b_{2}\right)+\ldots$ This is a global, across-stimuli concept and is the cumulative probability analog of PS (Equation 2). It is also linked to PS via Theorem 5 in Ashby and Townsend (1986,p. 166), which states that if PS and DS hold for two dimensions, then MRI is logically implied for those dimensions.

The second probability-based definition of independence of two (or more) dimensions within a given stimulus is sampling independence (SI). Again, it is the cumulative probability analog of PI (Equation 1) and is defined to hold in a given stimulus, $S_{r}$, when the probability of reporting the joint event of the two components equals the product of the marginal probabilities of reporting each component individually:

$$
P\left(a_{i} b_{j} \mid S_{r}\right)=P\left(a_{i} \mid S_{r}\right) P\left(b_{j} \mid S_{r}\right) .
$$

Theorem 1 in Ashby and Townsend (1986, p. 160) states that if DS is assumed to hold, SI logically implies PI in a given stimulus.

The second set of behaviorally defined measures that can be used to test PI and the two separabilities are the $d^{\prime}$ and $\beta$ parameters of signal detection theory. There are two types of signal detection parameters that can be defined in multidimensional perceptual space: marginal and conditional. Both are estimated, as in Green and Swets (1966), from the probability of a "hit" (here we arbitrarily define a hit as level 2 on a dimension when level 2 was presented to the subject) and the probability of a "false alarm" (when the subject responds level 2 when in fact level 1 was presented). The difference lies in that the marginal parameters (and estimates) use the marginal densities (and cumulative probabilities), whereas the conditional estimates are found from densities resulting after conditioning on a particular level of the second dimension. For example, we define conditional $d^{\prime}$ and $\beta$ between the two shapes, given that the subject correctly responded "red," and, similarly, we define $d^{\prime}$ and $\beta$ between the shapes, given the subject's incorrect response that he/she saw "red." This can be carried out for each dimension, conditioned on each type of response to the other dimension. Further details and more technical definitions may be obtained from Kadlec and Townsend (1992a, 1992b). 


\section{TESTS OF PS, DS, AND PI}

We have recently shown that the two types of signal detection parameters are related to PI, PS, and DS (Kadlec \& Townsend, 1992a) and thereby contribute to a stronger empirical test of PI, PS, and DS. These theoretical results, in addition to those of Ashby and Townsend (1986), have been used to develop a methodology that uses all the observable conditions - MRI, SI, and the two sets of signal detection estimates-to test PS, DS, and PI of two or more perceptual dimensions (Kadlec \& Townsend, 1992a, 1992b). This technique is called multidimensional signal detection analyses (MSDA).

Ashby and Lee (1991) have also developed a technique, based on hierarchical model-fitting, to test for PS, DS, and PI in the context of GRT. In this procedure, a series of nested models is fit to the data until no significant improvement in fit is found. For example, to infer that PS holds for dimension $A$ (and DS and PI hold), a model assuming PS, DS, and PI is tested against a model where DS and PI hold but PS for dimension $A$ does not; if no significant improvement is found, PS for dimension $A$ is supported. This is a valuable technique and provides a complementary analysis to the one presented here. Compared with MSDA, it is computationally much more intensive and is limited by the degrees of freedom available from the confusion data - a limitation that is especially crucial in designs with small confusion matrices. ${ }^{1}$

In MSDA, the theoretical relationships between all the observable conditions and PS, DS, and PI lead directly to inferences about these types of dimensional interaction. If we list all possible experimental outcome combinations, we can find their consequences (these are summarized in Tables 1 and 2). In the marginal analyses, or macroanalyses (Table 1), a given pattern of results in tests of MRI, marginal $d^{\prime}$, and marginal $\beta$-where $\mathrm{T}$ (true) indicates that the condition holds in the data, and $\mathrm{F}$ ( false) means it has been violated by the data--allows inferences about PS and DS. Table 2 shows the microanalyses, where tests of SI of stimulus dimensions within each stimulus, as well as

Table 1

Inferences From Macroanalyses About PS and DS From Observable Conditions

\begin{tabular}{ccccc}
\hline & $\begin{array}{c}\text { Observed Results } \\
\text { for One Dimension }\end{array}$ & & \multicolumn{2}{c}{$\begin{array}{c}\text { Conclusions } \\
\text { for One Dimension }\end{array}$} \\
\cline { 3 - 6 } MRI? & $\begin{array}{c}\text { Marginal } \\
d^{\prime} \text { equal? }\end{array}$ & $\begin{array}{c}\text { Marginal } \\
\beta \text { equal? }\end{array}$ & PS & DS \\
\hline$T$ & $\mathrm{~T}$ & $\mathrm{~T}$ & $\mathrm{y}$ & $\mathrm{y}$ \\
$\mathrm{T}$ & $\mathrm{T}$ & $\mathrm{F}$ & $\mathrm{y}$ & $\mathrm{N}$ \\
$\mathrm{T}$ & $\mathrm{F}$ & $\mathrm{T}$ & $\mathrm{N}$ & $\mathrm{y}$ \\
$\mathrm{T}$ & $\mathrm{F}$ & $\mathrm{F}$ & $\mathrm{N}$ & $\mathrm{N}$ \\
$\mathrm{F}$ & $\mathrm{T}$ & $\mathrm{T}$ & $\mathrm{y}$ & $? \mathrm{n}$ \\
$\mathrm{F}$ & $\mathrm{T}$ & $\mathrm{F}$ & $\mathrm{y}$ & $\mathrm{N}$ \\
$\mathrm{F}$ & $\mathrm{F}$ & $\mathrm{T}$ & $\mathrm{N}$ & $?$ \\
$\mathrm{~F}$ & $\mathrm{~F}$ & $\mathrm{~F}$ & $\mathrm{~N}$ & $?$ \\
\hline
\end{tabular}

Note - MRI, marginal response invariance; PS, perceptual separability; DS, decisional separability; T, true; F, false; y, (weak) yes; N, no; ?y, unknown but possibly yes; ?n, unknown but possibly no; ?, unknown. This table is adapted from Kadlec and Townsend's (1992b) Table 1.
Table 2

Inferences From Microanalyses About PI and DS From Observable Conditions

\begin{tabular}{cccccc}
\hline & $\begin{array}{c}\text { Observed Results } \\
\text { for a Pair of Stimuli }\end{array}$ & & \multicolumn{2}{c}{$\begin{array}{c}\text { Conclusions } \\
\text { for a Pair of Stimuli }\end{array}$} \\
\cline { 5 - 6 } SI? & $\begin{array}{c}\text { Conditional } \\
d^{\prime} \text { equal? }\end{array}$ & $\begin{array}{c}\text { Conditional } \\
\beta \text { equal? }\end{array}$ & & PI & DS \\
\hline T or F & $\mathrm{T}$ & $\mathrm{T}$ & $\mathrm{y}$ & $\mathrm{y}$ \\
$\mathrm{T}$ & $\mathrm{T}$ & $\mathrm{F}$ & $? \mathrm{y}$ & $\mathrm{N}$ \\
$\mathrm{T}$ & $\mathrm{F}$ & $\mathrm{T}$ & $? \mathrm{y}$ & $\mathrm{N}$ \\
$\mathrm{T}$ & $\mathrm{F}$ & $\mathrm{F}$ & $? \mathrm{y}$ & $\mathrm{N}$ \\
$\mathrm{F}$ & $\mathrm{T}$ & $\mathrm{F}$ & $? \mathrm{n}$ & $? \mathrm{n}$ \\
$\mathrm{F}$ & $\mathrm{F}$ & $\mathrm{T}$ & $? \mathrm{n}$ & $? \mathrm{n}$ \\
$\mathrm{F}$ & $\mathrm{F}$ & $\mathrm{F}$ & $? \mathrm{n}$ & ?n \\
\hline
\end{tabular}

Note-SI, sampling independence; PI, perceptual independence; DS, decisional separability; T, true; F, false; y, (weak) yes; N, no; ?y, unknown but possibly yes; ?n, unknown but possibly no. This table is adapted from Kadlec and Townsend's (1992b) Table 2.

estimates of conditional $d^{\prime} \mathrm{s}$ and $\beta \mathrm{s}$, yield inferences about PI and DS.

In the Conclusions columns, "N" $(n o)$ indicates a strong conclusion that the effect was logically falsified, whereas " $y$ " (yes) denotes weak support for the conclusion insofar as the observed results are consistent with the conclusion but are not a logical implication. This stems from the form of the theoretical relationships where the logical implications of the theorems relating the SDT parameters with PS, DS, and PI are unidirectional - that is, where the logical implications are from the unobservables (PS, DS, and PI) to the observable conditions (MRI, SI, equality of marginal or conditional $d^{\prime}$ and $\beta$ ). For example, one theorem states that if PI and DS hold, then the conditional $d^{\prime}$ and $\beta$ parameters will be equal; however, the contrapositive statement has not been proved. Using this theorem, if the conditional estimates are found to be equal, such results are consistent with PI and DS but are not logically implied. In such cases, we say that PI and DS have found weak support (denoted by "y"; see Kadlec \& Townsend, 1992a, for further discussion on this point).

Furthermore, because most of the theoretical relationships are unidirectional, some combinations of results do not yield a conclusive inference. These cases, which appear particularly in the microanalyses, while inconclusive are nevertheless suggestive in the direction indicated. This is denoted by prefixing the conclusion with "?" and merely indicates that the conclusions should be interpreted with caution.

The software for computing MRI, SI, and the signal detection estimates, along with the corresponding inferences, is described next.

\section{MSDA PROGRAM}

\section{Specification of Stimulus Design}

Recall that the feature-complete factorial design of the stimulus set is a factorial combination of the levels on each of the dimensions. For example, for testing three dimensions, $A, B$, and $C$, with two levels on dimension $A$, three levels on dimension $B$, and four levels on dimen- 
sion $C$, the design is specified as a $2 \times 3 \times 4$. The order of the dimensions is important here for the subsequent analyses, since the stimulus design specifies how the stimuli are labeled. For example, a $2 \times 3$ design has stimuli coded as $11,12,13,21,22,23$, which is different from a $3 \times 2$ design whose stimuli are $11,12,21,22,31,32$. (Note that analysis of both $2 \times 3$ and $3 \times 2$ designs would require a $6 \times 6$ confusion matrix.)

The program can handle up to four dimensions and up to four levels on each dimension. Experiments with more dimensions and levels would not be practically feasible; thus, these upper limits seem sufficient. A $4 \times 4 \times 4 \times 4$ design, however, would require a $512 \times 512$ confusion matrix, which would not be encountered in practice; thus, a limitation is placed on the size of the confusion matrix, and it must be less than $50 \times 50$. This combination of values provides flexibility for both the number of dimensions and the number of levels employed in a given study. For example, studies with four dimensions, each with two levels, have been performed (e.g., Townsend, $\mathrm{Hu}, \&$ Kadlec, 1988). Two-dimensional studies where each dimension had four levels have also been reported (e.g., Nosofsky, 1985).

The minimum number of dimensions is two, and the minimum number of levels per dimension is also two. Thus, the smallest design is a $2 \times 2$, requiring a $4 \times 4$ confusion matrix for analysis.

\section{Input Confusion Matrix}

As noted above, the maximum size of the input confusion matrix is $50 \times 50$, and the minimum is $4 \times 4$. The frequency of each of the possible responses to a given stimulus presented is tabulated in one row of the confusion matrix. The diagonal entries of the matrix contain the frequencies of the correct responses.

Table 3 shows an example of an $8 \times 8$ confusion matrix obtained from a recent study (Kadlec, 1992) where three dimensions (CURVATURE, ORIENTATION, and LOCATION) were each presented at one of two levels in a $2 \times 2 \times 2$ design. The stimuli were arcs of two curvatures, smaller (level 1) and larger (level 2), with attached radial lines of two orientations, $50^{\circ}$ and $55^{\circ}$ (levels 1 and 2 , respectively), similar to stimuli constructed of circles or semicircles of different diameters with embedded radial lines employed by Shepard (1964) and Nosofsky (1985), among others. Furthermore, each stimulus was presented to the observer

Table 3

An $8 \times 8$ Confusion Matrix Obtained From a $2 \times 2 \times 2$ Feature-Complete Factorial Design

\begin{tabular}{crrrrrrrr}
\hline & \multicolumn{7}{c}{ Response } \\
\cline { 2 - 10 } Stimulus & 111 & 112 & 121 & 122 & 211 & 212 & 221 & 222 \\
\hline 111 & 84 & 1 & 6 & 0 & 7 & 0 & 2 & 0 \\
112 & 10 & 77 & 0 & 7 & 1 & 3 & 0 & 2 \\
121 & 42 & 2 & 47 & 1 & 4 & 0 & 4 & 0 \\
122 & 13 & 25 & 20 & 30 & 5 & 3 & 2 & 2 \\
211 & 1 & 0 & 0 & 0 & 74 & 1 & 24 & 0 \\
212 & 1 & 0 & 0 & 2 & 22 & 59 & 1 & 15 \\
221 & 1 & 0 & 7 & 0 & 20 & 3 & 57 & 12 \\
222 & 1 & 0 & 2 & 6 & 7 & 24 & 16 & 44 \\
\hline
\end{tabular}

at one of two different locations. The analysis of this confusion matrix will be used in the remainder of this report as an example. As Table 3 shows, the input file for this 2 $\times 2 \times 2$ design contains an $8 \times 8$ matrix of integer-valued frequencies.

\section{Main Menu}

The main menu is shown in Appendix A. Option 0 gives a brief overview of the design limits and the format of the input confusion matrix, as described above. Option 1 allows the user to define the design that will be used in the analyses. In selecting Option 1, the user is prompted by a series of queries. First, the number of dimensions must be specified. Second, for each dimension, the user may optionally provide a name/label for the dimension but must specify the number of levels. The program then computes the size of the confusion matrix required for the analysis and provides a summary of the design. If the wrong design has been entered, the user can simply return to the main menu and select Option 1 again.

Option 2 prompts the user to enter the name of the input file containing the confusion frequencies. The name should be a standard file name, with eight alphanumeric characters and an optional three character extension. If the input file name has the extension ".MTX", all such files found in the current directory are listed for the user's convenience. This ".MTX" extension, however, is not necessary, and any file name can be entered.

The default name of the output file is generated from the input file name without the extension and by adding the extension ".MSD". Alternatively, the user can specify her/ his own name for the output file.

After specifying the design by Option 1 and the file name of the input confusion matrix in Option 2, the confusion matrix can be viewed on the screen by selecting Option 3 . This provides a convenient check to make sure that the matrix has been read in correctly.

Option 4 runs the analysis of the confusion matrix. The analysis and sample output are described in the next section. The output file can be viewed onscreen or printed by selecting Option 5 or printed by selecting Option 6. To exit the program, Option $\mathrm{x}$ is selected.

\section{Estimation of Signal Detection Parameters}

The estimation takes place for each given dimension across levels of (or conditioning on) a secondary dimension at all fixed levels of the remaining dimension(s). The analysis is done for all dimensions combined in all possible and nonredundant ways. For example, for three dimensions there are 3 ! combinations of orders of the dimensions: $123,132,213,231,312$, and 322 . The order 123 tests dimension 1 across levels of (or conditional on) dimension 2 at all (fixed) levels of dimension 3.

The marginal $d$ 's and $\beta \mathrm{s}$ are estimated from the probabilities obtained from the confusion matrix. It is assumed that for all estimates, both the "noise" and "signal+noise" distributions are standard normal, since in the present paradigm, we cannot estimate the variances of the distributions. For every estimate, the $z$ values corresponding to the 
probability of "hit" (proportion of times that the observer responded with level 2 when level 2 was presented) and "false alarm" (proportion of times that the observer responded with level 2 when level 1 was presented) are then employed to compute the $d^{\prime}$ and $\beta$ estimates, as in standard signal detection theory.

In the case of conditional $d^{\prime} \mathrm{s}$ and $\beta \mathrm{s}$, estimation is conducted similarly, once the appropriate probabilities of "hit" and "false alarm" are obtained. To compute the probabilities of "hit" and "false alarm" conditional on the subject's response to a second dimension, the definition of conditional probability is used: For any two events denoted $E 1$ and $E 2, P(E 1 \mid E 2)=P(E 1$ and $E 2) / P(E 2)$. Thus, for example, the probability of "hit" on dimension $A$-which occurs when the observer responds level 2 on $A(a 2)$ when in fact $A$ was presented at level $2(A 2)$-conditional on, say, the "hit" on dimension $B$, is given by $P\left(a_{2} \mid b_{2}\right.$ and $\left.A_{2} B_{2}\right)=P\left(a_{2}\right.$ and $\left.b_{2} \mid A_{2} B_{2}\right) / P\left(b_{2} \mid A_{2} B_{2}\right)$; the probability of "false alarm" on $A$, conditional on the "hit" on $B$ is $P\left(a_{2} \mid b_{2}\right.$ and $\left.A_{1} B_{2}\right)=P\left(a_{2}\right.$ and $\left.b_{2} \mid A_{1} B_{2}\right) / P\left(b_{2} \mid A_{1} B_{2}\right)$.

To obtain the $z$ values for the normal distribution, a "table look-up" algorithm is used, with interpolation if necessary.

\section{Output of MSDA and Its Interpretation}

Appendix $\mathrm{B}$ shows the output obtained for the confusion matrix shown in Table 3 analyzed in a $2 \times 2 \times 2$ stimulus design. The marginal results are reported first, with MRI probabilities, and marginal $d^{\prime}$ s and $\beta$ s tested for equivalence, as described below. This is followed by the conditional analyses, tests of SI followed by the conditional signal detection microanalyses. The conclusions regarding PS, DS, and PI are also reported. For example, if the marginal $d$ 's are equivalent, support for PS is inferred (Table 1).

In the marginal analyses, conclusions about PS and DS are reported for each dimension across the levels of the other dimension. Failure of either type of separability is indicated by the $\sim($ logical not) sign in front of the corresponding abbreviation. Furthermore, if PS has failed ( $\sim \mathrm{PS})$, the direction of the interaction can also be immediately inferred from the relative sizes of the reported marginal $d^{\prime}$ estimates. For example, in Appendix B, CURVATURE failed to be perceptually separable across levels of ORIENTATION at the second level of the LOCATION dimension. This is concluded because the marginal $d$ 's were significantly different, with the $d^{\prime}$ at the first level of ORIENTATION being larger $\left(d^{\prime}=3.45\right)$ than at the second level of ORIENTATION $\left(d^{\prime}=2.52\right)$. This means that when the lines were oriented at $50^{\circ}$ (level 1 ), the curvatures were more easily discriminable (because the $d^{\prime}$ is larger) than with $55^{\circ}$ lines (level 2). ${ }^{2}$

The conditional analyses are reported next, beginning with tests of SI. For SI, in designs with three or four dimensions, the highest order SI is tested first. For example, in a three-dimensional design, three-way SI is tested first: if three-way SI holds, two-way SI tests are not performed since it follows that if three-way SI holds, all subordinate two-way SI tests will also hold. However, if three-way SI fails for a particular stimulus-response cell of the confu- sion matrix, all possible two-way SI tests are conducted to isolate which pair or pairs of dimensions violated SI. For two-dimensional designs, no further tests are necessary.

The conditional signal detection analyses are reported last. As with the marginal analyses, these involve all possible combinations of dimensions. In this case, tests are always performed pairwise for estimates conditional on adjacent levels of the secondary dimension: for example, estimates for dimension $A$ conditioning on level 1 versus level 2 on dimension $B$. In the case of more than two levels on the secondary dimension, conditional tests are also conducted on all other adjacent levels--that is, conditioning on (1) level 1 versus level 2, (2) level 2 versus level 3, and (3) level 3 versus level 4 (see example in Appendix C, which is discussed further below).

The marginal signal detection estimates are again shown in the columns labeled "Marg." for comparison purposes; however, these marginal values are not included in the significance tests for conditional signal detection estimates. The conclusions about PI and DS are reported. Note that in the conditional signal detection tests, the test for PI is always for a given pair of stimuli. For example, in Appendix $\mathrm{B}$, the conditional $d^{\prime}$ and $\beta$ for CURVATURE conditional on LOCATION (at level 2), with ORIENTATION fixed at level 1 , are equal. In other words, conditioning on the subject's responses above and below the LOCATION criterion results in equal $\beta \mathrm{s}$ and $d^{\prime}$ s for CURvature $\left(d^{\prime}=3.064\right.$ below the LOCATION criterion where the subject incorrectly responds level 1 , and $d^{\prime}=3.538$ above the LOCATION criterion where the subject correctly responds level 2). This means that the data are consistent with perceptual independence between CURVATURE and LOCATION in stimuli $C_{1} O_{1} L_{2}$ and $C_{2} O_{1} L_{2}$.

When PI has failed-a conclusion that cannot be strongly inferred when DS has failed but is consistent with the observed results (denoted as ? PI in the output) - the direction of the interaction can again be inferred by examining the relative sizes of the conditional $d^{\prime}$ estimates. For example, CURVATURE and ORIENTATION (at level 1) are not perceptually independent (see, e.g., at the second level of LOCATION), because the CURVATURE $d^{\prime}$, conditioned on the subject's correct identification of ORIENTATION as being at the first level $\left(d^{\prime}=3.97\right)$, is significantly larger than the $d^{\prime}$ conditioned on the subject's incorrect identification of ORIENTATION at second level $\left(d^{\prime}=1.985\right)$. This tests PI between CURVATURE and ORIENTATION in the stimuli $C_{1} O_{1} L_{2}$ and $C_{2} O_{1} L_{2}$ (with LOCATION at level 2), and the unequal $d^{\prime}$ 's indicate that at least one of the equal density contours that correspond to these two stimuli is a tilted ellipse, such that the distance between the densities below the ORIENTATION criterion (level 1) is larger than the distance above the ORIENTATION criterion (level 2) (see Figure 2).

As an example of how the information from the marginal and conditional analyses can be combined into a graphical representation of the stimuli in a perceptual space, a view of the equal density contours for CURVATURE and ORIENTATION, at the second level of LOCATION, is shown in Figure 2 for these confusion data. This repre- 


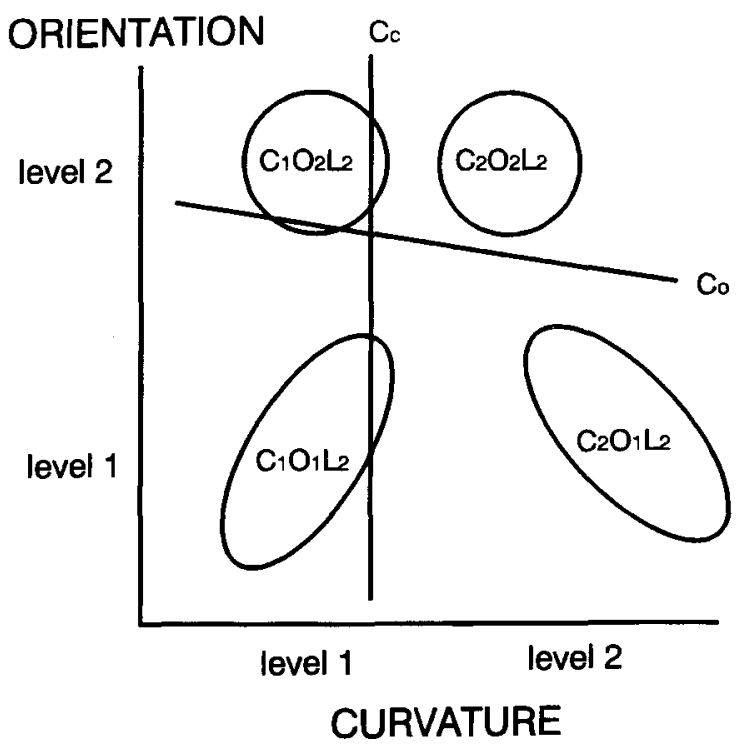

Figure 2. Equal density contours for CURVATURE and ORIENTATION, at the second level of LOCATION, for the confusion data in Table 3 that have been analyzed as a $2 \times 2 \times 2$ stimulus design (results given in Appendix B). $C_{o}=$ decision criterion for ORIENTATION; $C_{c}=$ decision criterion for CURVATURE. See text for further discussion.

sentation captures the following features of the analyses: (1) The marginal $d^{\prime}$ for CURVATURE is larger at the first level of ORIENTATION (3.45) than at the second level (2.52), indicating failure of PS of CURVATURE across ORIENTATION. This is indicated by the different distances on the CURVATURE axis (which are proportional to the $d$ 's) between the centers of the ellipses (i.e., the means of these densities). (2) The marginal $d$ 's are equal for ORIENTATION across CURVATURE ( 1.44 and 1.383 ) again because the distances between the centers in this case are equal on the ORIENTATION axis. (3) DS, which was inconclusive for both dimensions in the marginal analyses, is supported for CURVATURE acroSS ORIENTATION and fails for ORIENTATION across CURVATURE in the microanalyses. (4) The circular contours of stimuli $\mathrm{C}_{1} \mathrm{O}_{2} \mathrm{~L}_{2}$ and $\mathrm{C}_{2} \mathrm{O}_{2} \mathrm{~L}_{2}$ allow for equal conditional $d$ 's, and with DS for CURVATURE (the $C_{c}$ criterion parallel to the ORIENTATION axis), equal conditional $\beta$ s. (5) The equal density contours for stimulus $C_{1} O_{1} L_{2}$ shows a positive dependence between CURVATURE and ORIENTATION, as shown by the positively tilted elliptical contour. This, along with the circular contour for stimulus $C_{1} O_{2} L_{2}$, results in a larger conditional $d^{\prime}$ to the left of the CURVATURE criterion, $C_{c}$, than the conditional $d^{\prime}$ to the right of this criterion. (6) The negatively tilted elliptical contour of stimulus $\mathrm{C}_{2} \mathrm{O}_{1} \mathrm{~L}_{2}$ is consistent with a smaller conditional $d^{\prime}$ to the left of the CURVATURE criterion. Further details about graphically representing stimulus configurations as equal density contours from these data analyses can be obtained from Kadlec and Townsend (1992a, 1992b).

Occasionally, it may occur that the analysis will yield a negative $d^{\prime}$ estimate. This has occurred in the present mi- croanalysis in the case of ORIENTATION conditional on LOCATION at level 1 of CURVATURE. It has been my experience that this occurs mostly in the microanalyses and that the magnitudes of such negative $d^{\prime}$ s are rather small $\left(\left|d^{\prime}\right|<0.5\right)$. Such small estimated values should be interpreted as $d$ 's of zero - that is, no discriminability between the two levels. If the magnitude of a negative $d^{\prime}$ estimate was "large," this would indicate that the elliptical densities are tilted in opposite directions and cross over each other, such that the $d^{\prime}$ above the decision criterion would have the opposite sign to the $d^{\prime}$ below the decision criterion.

It may be noted that an $8 \times 8$ confusion matrix is also consistent with $4 \times 2$ and $2 \times 4$ stimulus designs. For illustration purposes, the output for the $4 \times 2$ design is shown in Appendix C; this would be interpreted as four levels of a FORM dimension and two levels of LOCATION. (The format of the output for the $2 \times 4$ design would be similar.)

The output and interpretations of these designs are identical to designs with two levels, but now each dimension is tested across all levels of the secondary dimension. Usually, the levels would be ordered along a single dimension (which is not the case in the present example); thus, there would be little, if any, distributional overlap between, say, levels 1 and 3 , rendering the tests for conditional $d^{\prime}$ and $\beta$ estimates at level 1 versus level 3 (and by fiat level 4) of less practical importance. Nonadjacent levels are therefore not tested.

\section{Significance Tests}

Two general types of significance tests are employed: testing whether two probabilities are equivalent (for MRI and SI), and testing whether two (or more) $d^{\prime}$ and $\beta$ values are equivalent.

To test two probability values for equivalence, a $z$ test (see, e.g., Hays, 1988, p. 258) is employed. Two probabilities, $p 1$ and $p 2$, are passed to the procedure, and the $z$ value is computed by

$$
\begin{aligned}
& z=(p 1-p 2-.5 / n) / \sigma_{P}, \text { if } p 1>p 2, \\
& z=(p 1-p 2+.5 / n) / \sigma_{p}, \text { if } p 2>p 1,
\end{aligned}
$$

where $\sigma_{P}=\sqrt{ }[n(p)(1-p)]$, with $p$ the larger of $p 1$ or $p 2$, and $n$ is the number of trials used to estimate each $p 1$ and $p 2$. The procedure returns the probability that the null hypothesis is true $\left[P\left(H_{o}\right)\right]$. For MRI with more than two levels across the secondary dimension, the largest and smallest values are selected and tested for equivalence, and if these values are not significantly different, MRI is inferred to hold.

To test the $d^{\prime}$ and $\beta$ estimates for equivalence, the nonparametric test of Grier (1971) was employed. This testing procedure uses the probabilities of hits and false alarms, one set for each level of the second dimension (see Grier, 1971 , for details). In general, in the marginal case, the number of $d^{\prime}$ s and $\beta$ s that are tested for equivalence is equal to the number of levels of the secondary dimension. In the conditional case, the number that is tested is always two (e.g., conditioning on level 1 vs. level 2). The procedure 
computes a mean value, and each obtained value is compared with this mean to determine whether it is significantly different from it. The default significance levels for the $d^{\prime}$ s are greater than or equal to 1.0 standard error of the mean (SEM; denoted by * in the output) or greater than or equal to $1.5 S E M$ (given as ${ }^{* *}$ ). For $\beta$, the default values are greater than or equal to $0.10\left(^{*}\right)$ or greater than or equal to $0.15\left(^{* *}\right)$ of the mean $B^{\prime \prime}$ index (called Bindex in output; Grier, 1971).

\section{Availability}

The program is available for IBM-compatible personal computers. It can be obtained free of charge from the author in compiled form. Please specify diskette size (5.25 or 3.5 in.).

\section{REFERENCES}

Ashby, F. G., \& LeE, W. (1991). Predicting similarity and categorization from identification. Journal of Experimental Psychology: General, 120, 150-172.

AshBy, F. G., \& Townsend, J. T. (1986). Varieties of perceptual independence. Psychological Review, 93, 154-179.

GARNER, W. R. (1974). Information processing and structure. Hillsdale, $\mathrm{NJ}$ : Erlbaum.

GARNER, W. R., \& Felfoldy, G. L. (1970). Integrality of stimulus dimensions in various types of information processing. Cognitive Psychology, 1, 225-241.

Green, D. M., \& SwETs, J. A. (1966). Signal detection theory and psychophysics. New York: Wiley.

GrIER, J. B. (1971). Nonparametric indexes for sensitivity and bias: Computing formulas. Psychological Bulletin, 75, 424-429.

HaYs, W. L. (1988). Statistics (4th ed.). New York: Holt, Rinehart \& Winston.

KADLEC, H. (1992). Information processing of stimulus modules and dimensions in visual perception: Independence and interactions. Unpublished doctoral dissertation, Purdue University.

KADLEC, H., \& TOWNSEND, J. T. (1992a). Implications of marginal and conditional detection parameters for the separabilities and independence of perceptual dimensions. Journal of Mathematical Psychology, 36, 325-374.

Kadlec, H., \& Townsend, J. T. (1992b). Signal detection analyses of dimensional interactions. In F. G. Ashby (Ed.), Multidimensional models of perception and cognition (pp. 181-227). Hillsdale, NJ: Erlbaum.
Nosofsky, R. (1985). Overall similarity and the identification of separable-dimension stimuli: A choice model analysis. Perception \& Psychophysics, 38, 415-432.

Pomerantz, J. R., \& Pristach, E. A. (1988). Emergent features, attention, and perceptual glue in visual form perception. Journal of Experimental Psychology: Human Perception \& Performance, 15, 635-649.

ShePARD, R. (1964). Attention and the metric structure of the stimulus space. Journal of Mathematical Psychology, 1, 54-87.

Thurstone, L. L. (1927). A law of comparative judgment. Psychological Review, 34, 273-286.

Townsend, J. T., Hu, G. G., \& Kadlec, H. (1988). Feature sensitivity, bias, and interdependencies as a function of intensity and payoffs. Perception \& Psychophysics, 43, 575-591.

Townsend, J. T., \& LANDON, D. E. (1983). Mathematical models of recognition and confusion in psychology. Mathematical Social Sciences, 4, 25-71.

\section{NOTES}

1. For example, a design to test two dimensions, where each has only two levels, results in a $4 \times 4$ confusion matrix with 12 degrees of freedom, thus allowing estimation of at most 12 parameters. A full Gaussian model for this simplest design that would allow failures of PS, DS, and PI would have 22 parameters ( 6 parameters for the means, i.e., the $x$-and $y$-coordinates of the mean vectors for each of 3 stimuli, since without loss of generality stimulus, $A_{1} B_{1}$ can be set at the origin; 12 parameters in total for the variance-covariance matrix $-\sigma_{x}, \sigma_{y}, \rho_{x y}-$ for each of the four stimuli; and 4 parameters for the coordinates of the decision bounds -2 for each decision bound if one wants to test for DS). The full model that would reflect failures of PS, DS, and PI, therefore, is not testable using this procedure in this design.

2. It may seem peculiar that the three dimensions in this study often violate PS $(\sim \mathrm{PS})$ when these seem intuitively separable and when the two dimensions of curvature (or size) and line orientation have in fact been shown to be "śeparable" in Garner's sense (e.g., Garner \& Felfoldy, 1970), in the PS sense of GRT (Ashby \& Lee, 1991, using hierarchical model-fitting; Kadlec \& Townsend, 1992b, in reanalyzing Nosofsky's, 1985, data using MSDA), and using multidimensional scaling analysis (e.g., Nosofsky, 1985; Shepard 1964). In this study, however, the task was more difficult with the addition of a third dimension (location), which may have resulted in the dimensions now interacting. These results are currently under investigation (Kadlec, 1992, and ongoing further experimentation). Ultimately, this hypothesis and other hypotheses about which dimensions are separable are empirical questions; the results presented here show that we cannot (or should not) trust our intuition in these matters.

\section{APPENDIX A \\ Main Menu of MSDA}

MAIN MENU
Please select number from the options below
0 - program info
1 - select DESIGN
2 - select CONFUSION MATRIX file for analysis
3 - VIEW confusion matrix
5 - ANALYZE confusion matrix
6 - PRINT contents of OUTPUT file on screen
$x-$ EXIT program




\section{APPENDIX B}

Sample Output of MSDA:

Results for the Confusion Matrix in Table 3 Analyzed Within a $2 \times 2 \times 2$ Design

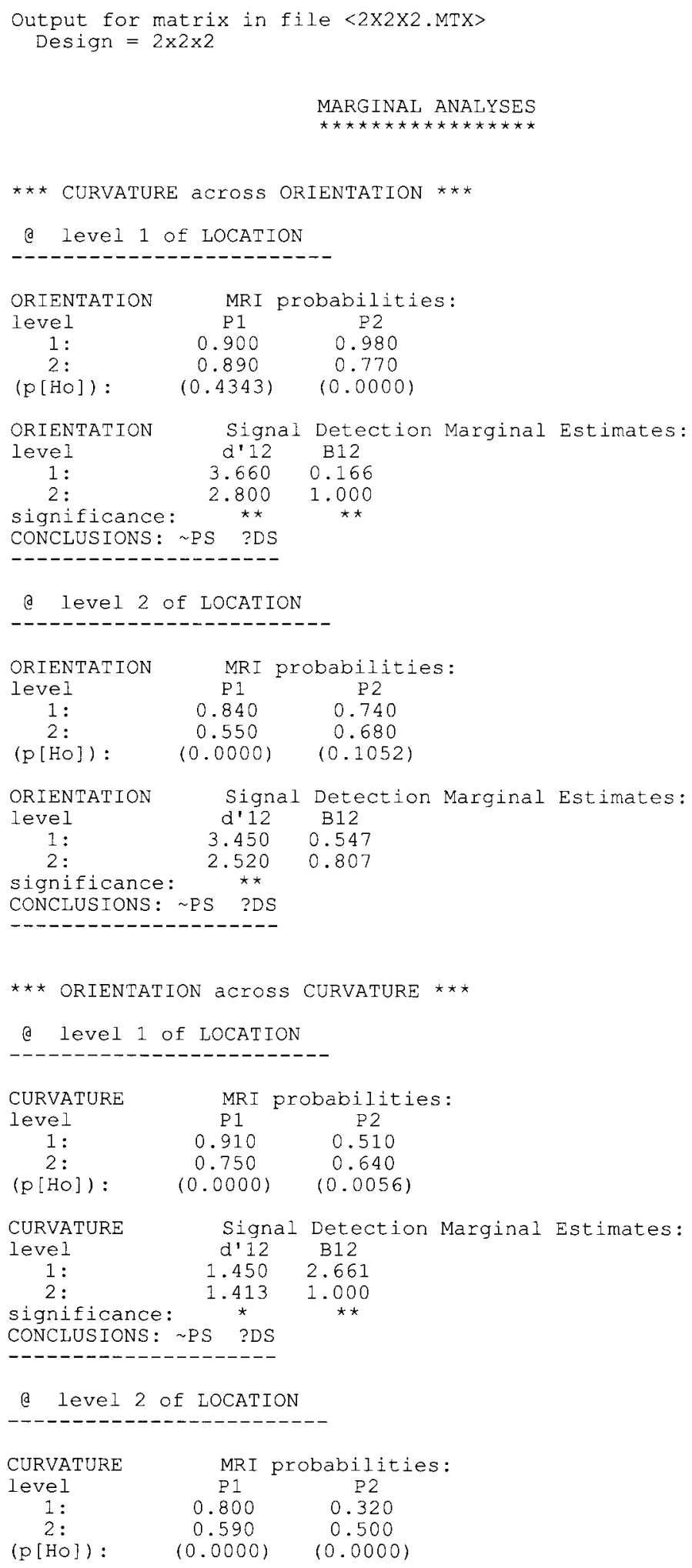




\section{APPENDIX B (Continued)}

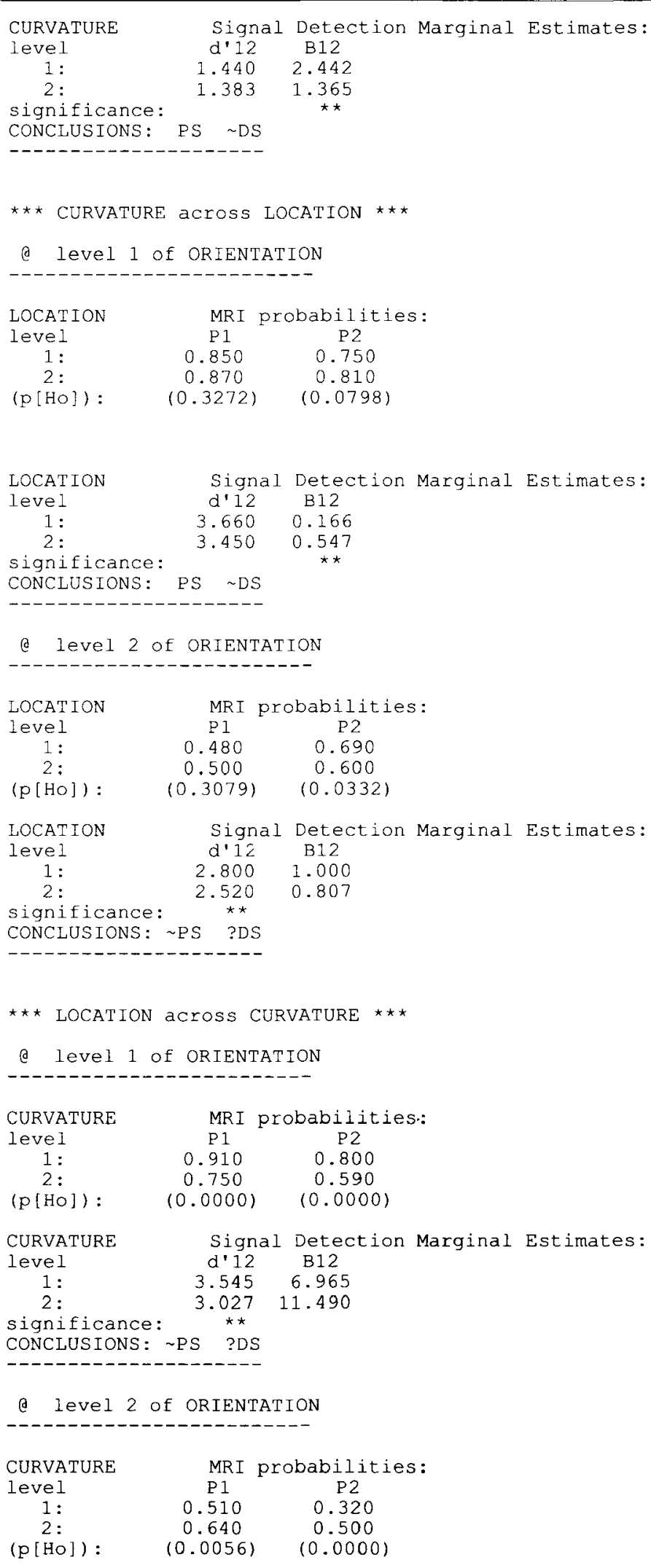




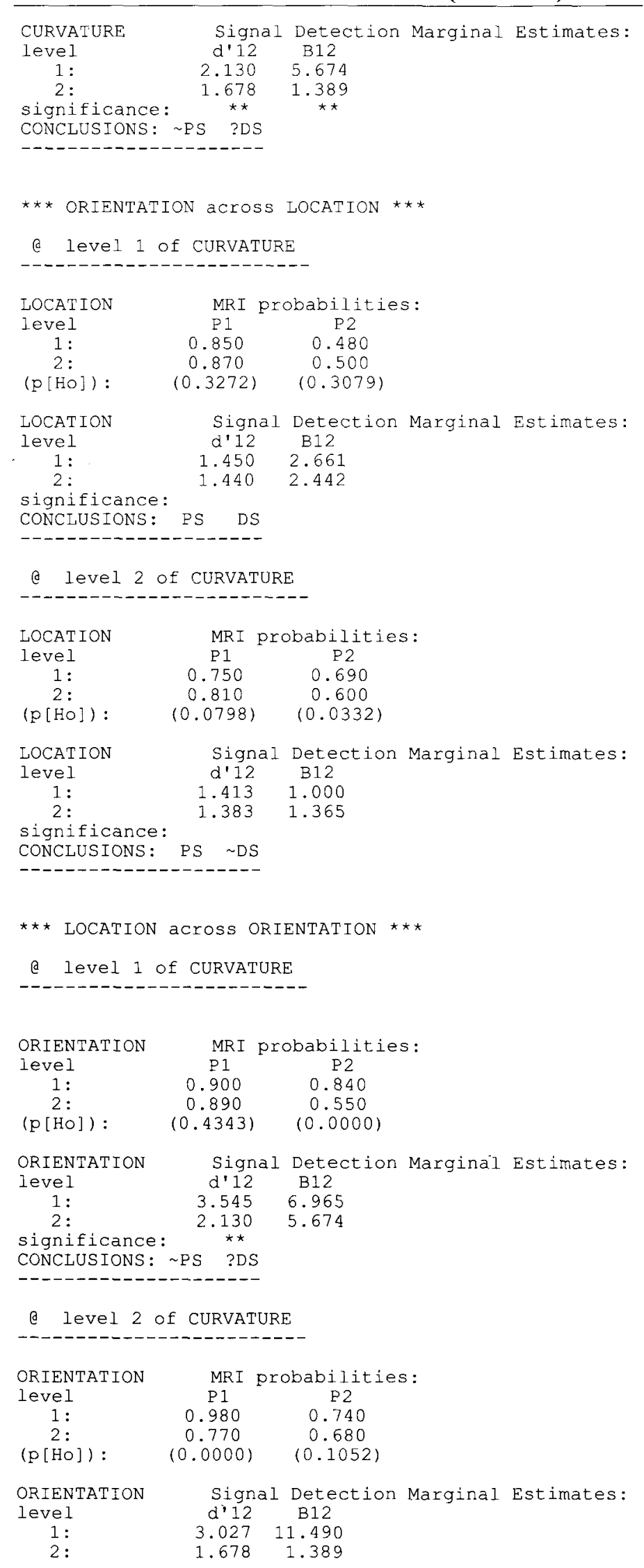




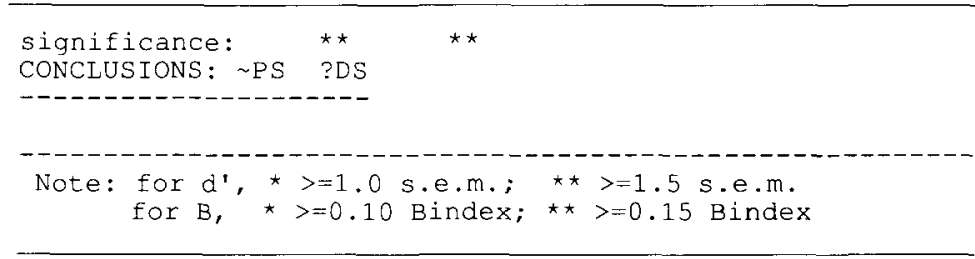

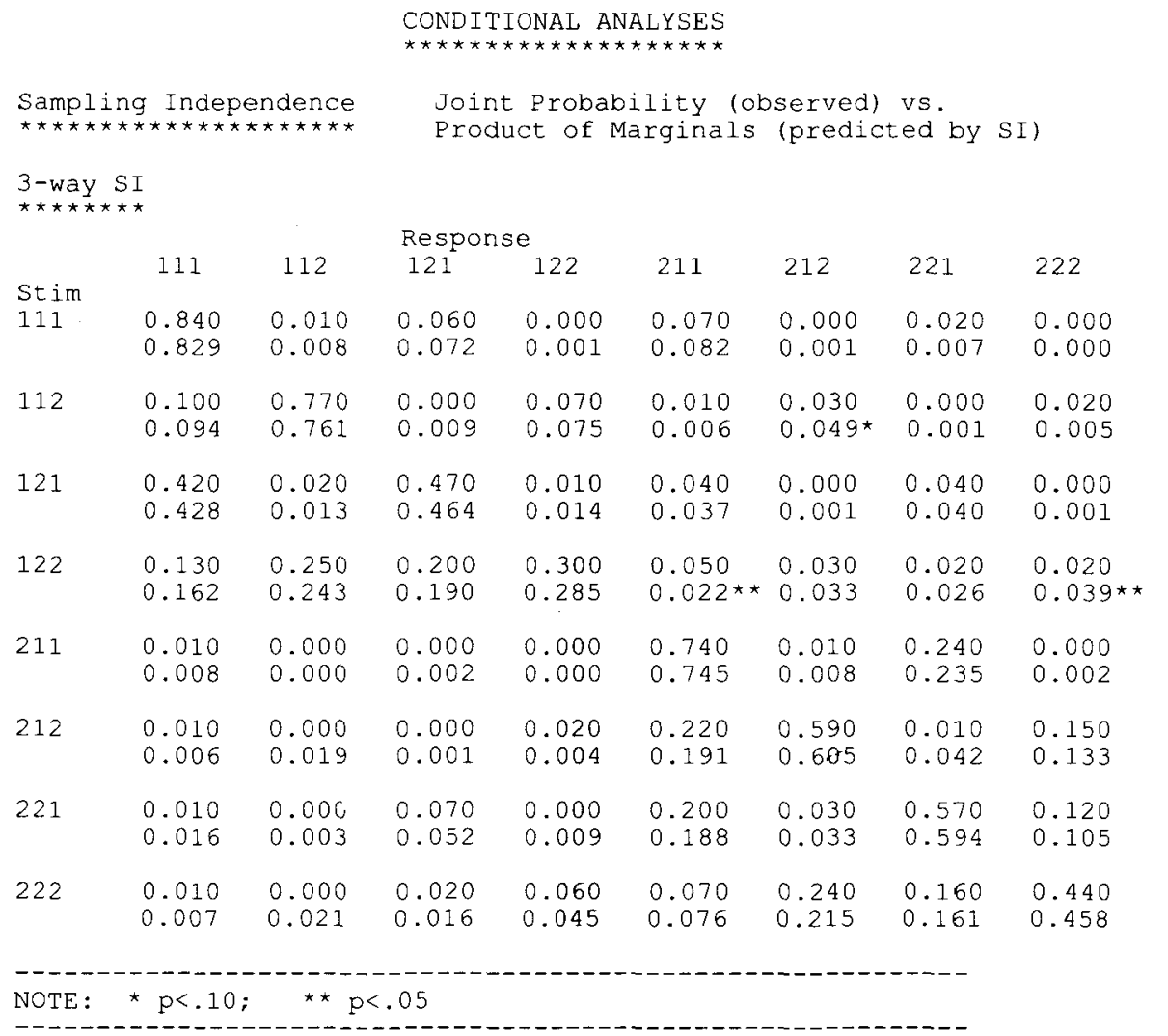

SUMMARY: sampling independence has failed in the following cells stimulus $=112$ response $=212$ stimulus $=122$ response $=211$ stimulus $=122$ response $=222$

Further tests of Sampling Independence

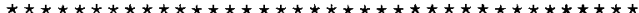

\begin{tabular}{llll} 
cel1 & \multicolumn{4}{c}{$\begin{array}{c}\text { joint } \\
\text { dimensions }\end{array}$} & $\begin{array}{c}\text { product } \\
\text { of marginals }\end{array}$ \\
1121212 & $1 \& 2$ & 0.040 & 0.055 \\
& $1 \& 3$ & 0.050 & 0.053 \\
1221211 & $2 \& 3$ & 0.800 & 0.810 \\
& $1 \& 2$ & 0.080 & $0.055^{\star}$ \\
1221222 & $1 \& 3$ & 0.070 & 0.048 \\
& $2 \& 3$ & 0.180 & 0.184 \\
& $1 \& 2$ & 0.040 & $0.065 \star$ \\
& $1 \& 3$ & 0.050 & 0.072 \\
& $2 \& 3$ & 0.320 & 0.324
\end{tabular}




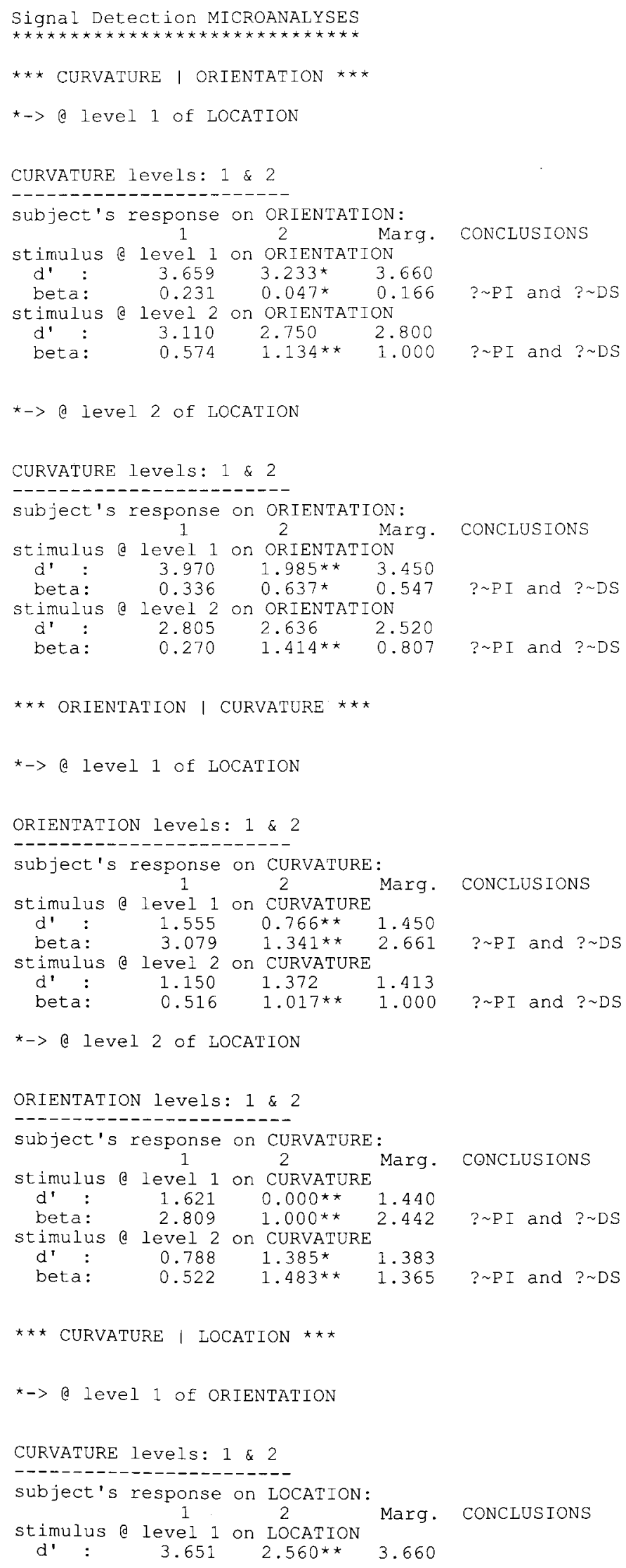




\section{APPENDIX B (Continued)}

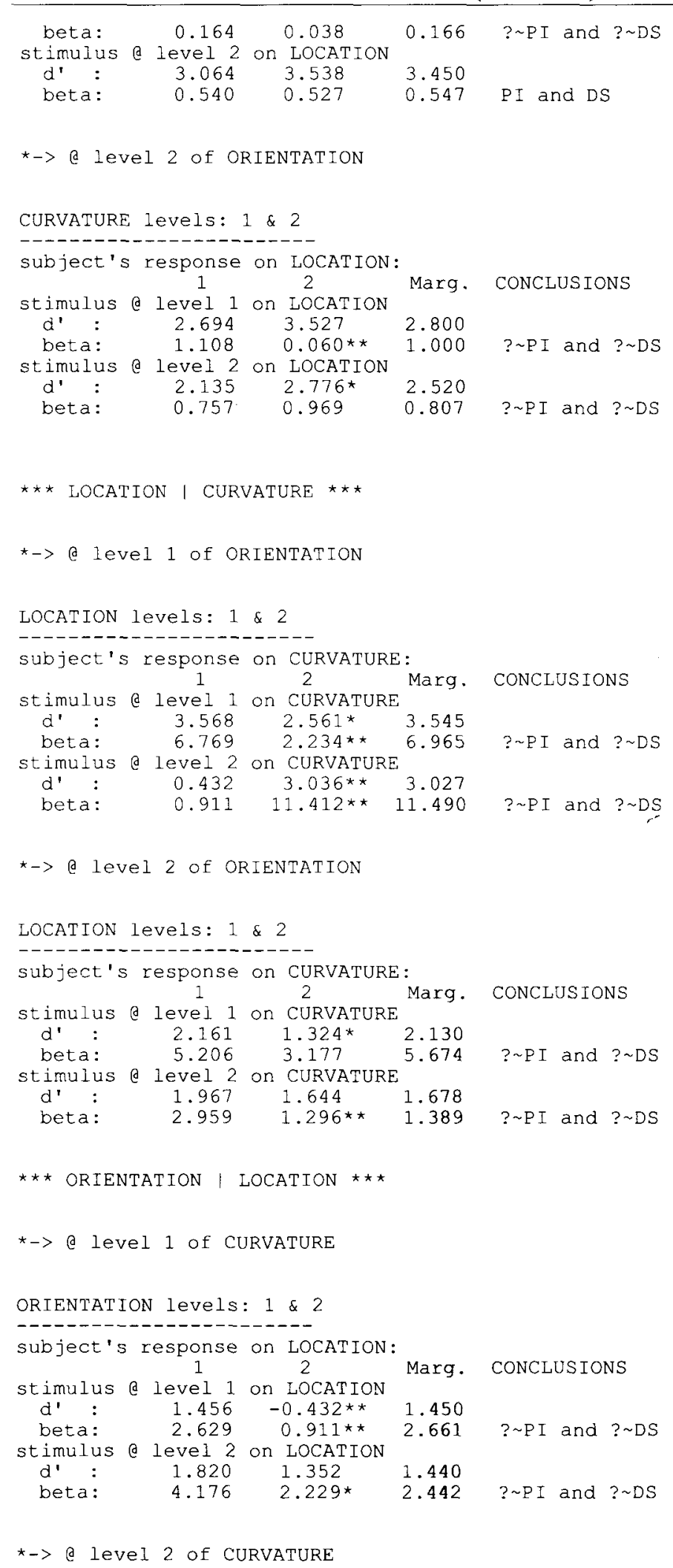


APPENDIX B (Continued)

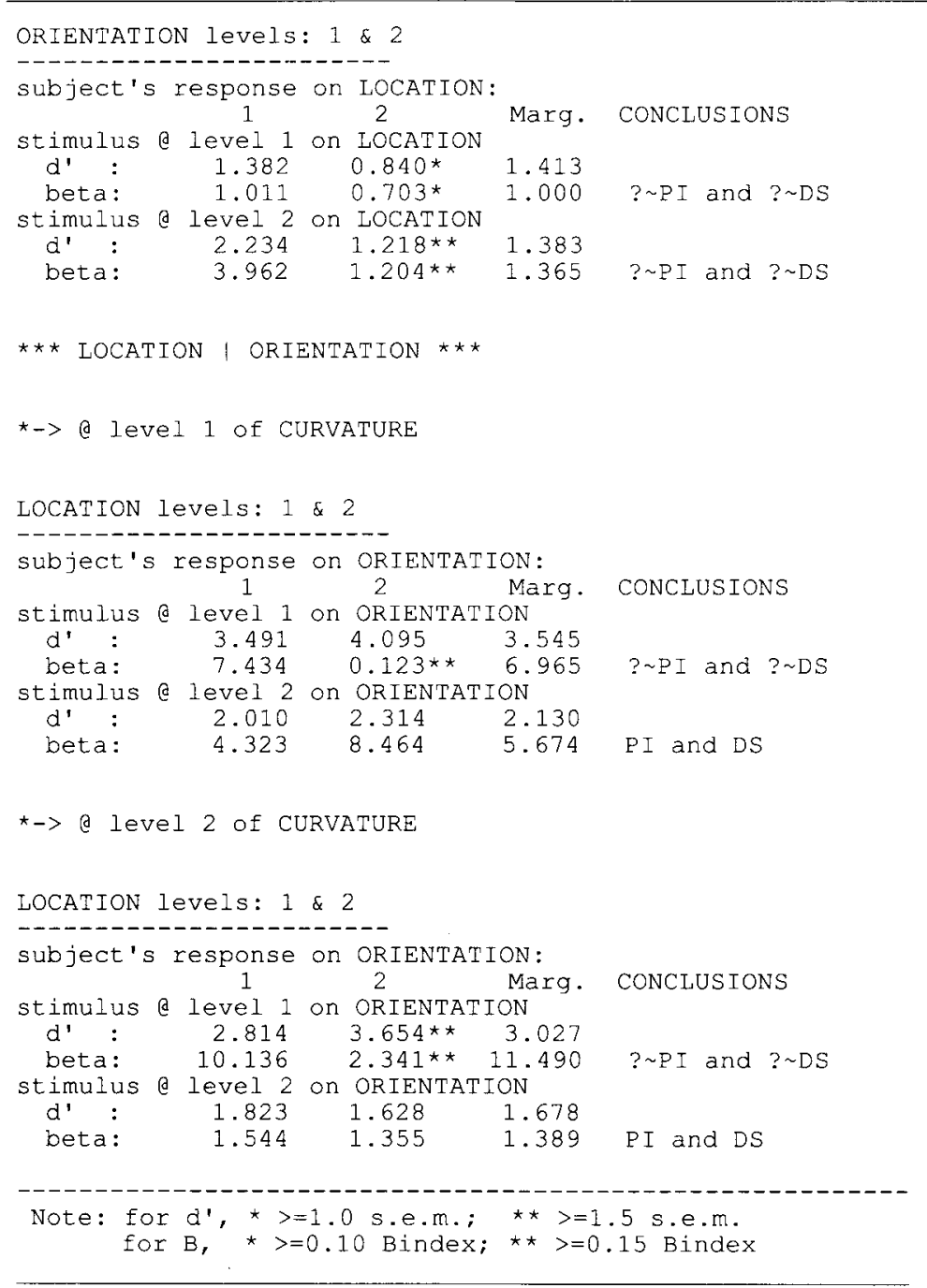

APPENDIX C

Sample Output of MSDA:

Results for the Confusion Matrix in Table 3 Analyzed Within a $4 \times 2$ Design

output for matrix in file <2X2X2. MTX>

Design $=4 \times 2$

MARGINAL ANALYSES

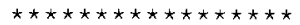

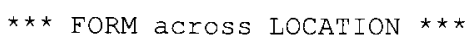

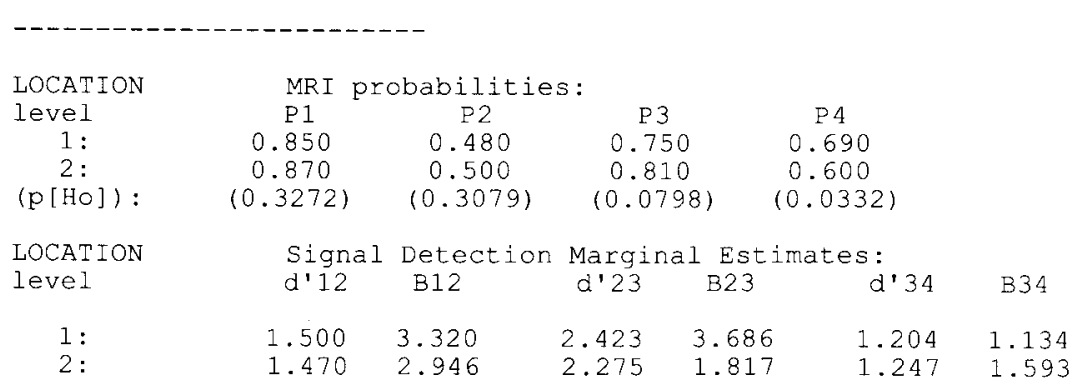




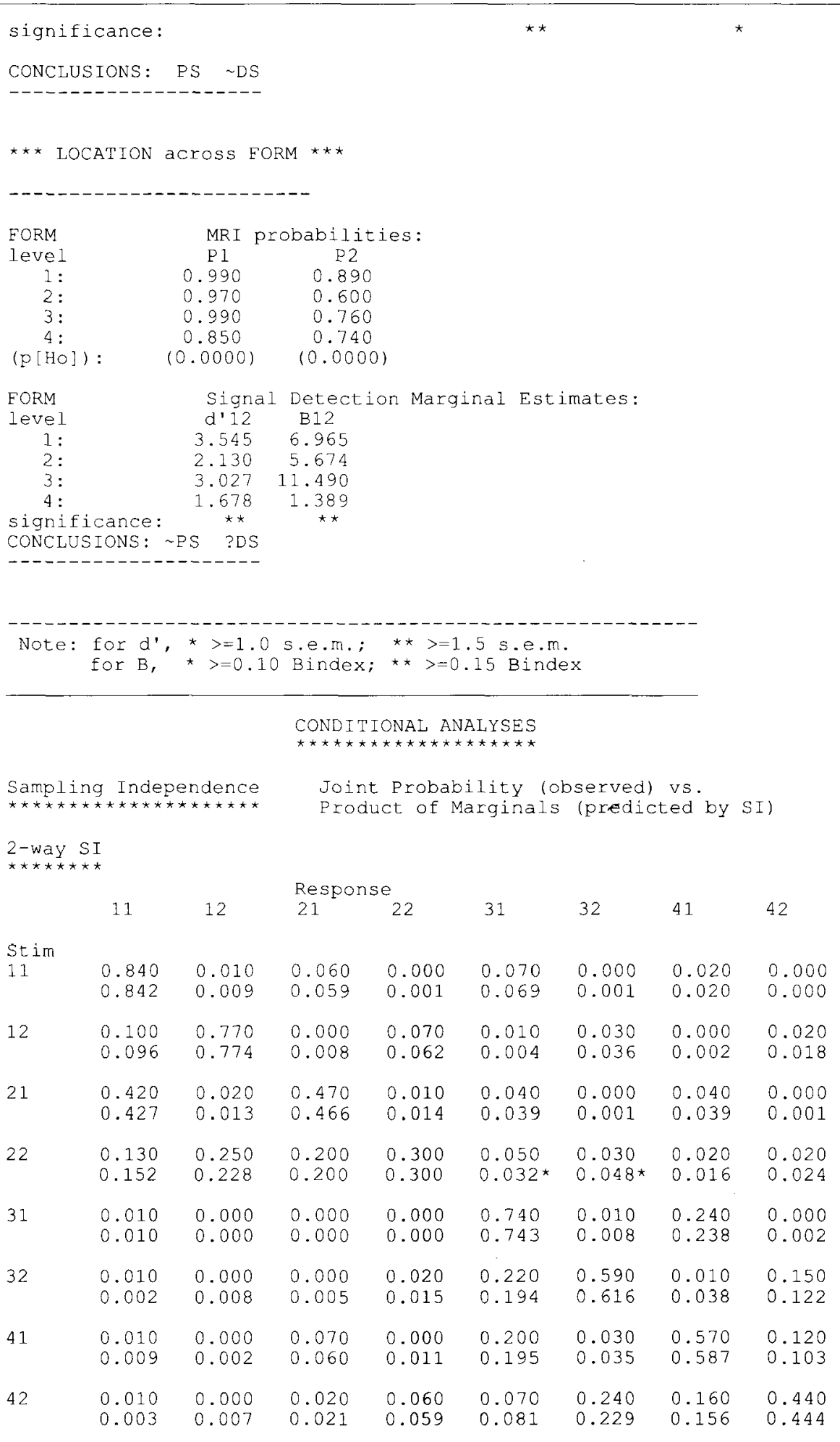

NOTE: * $\mathrm{p}<.10 ; \quad$ * $\mathrm{p}<.05$ 


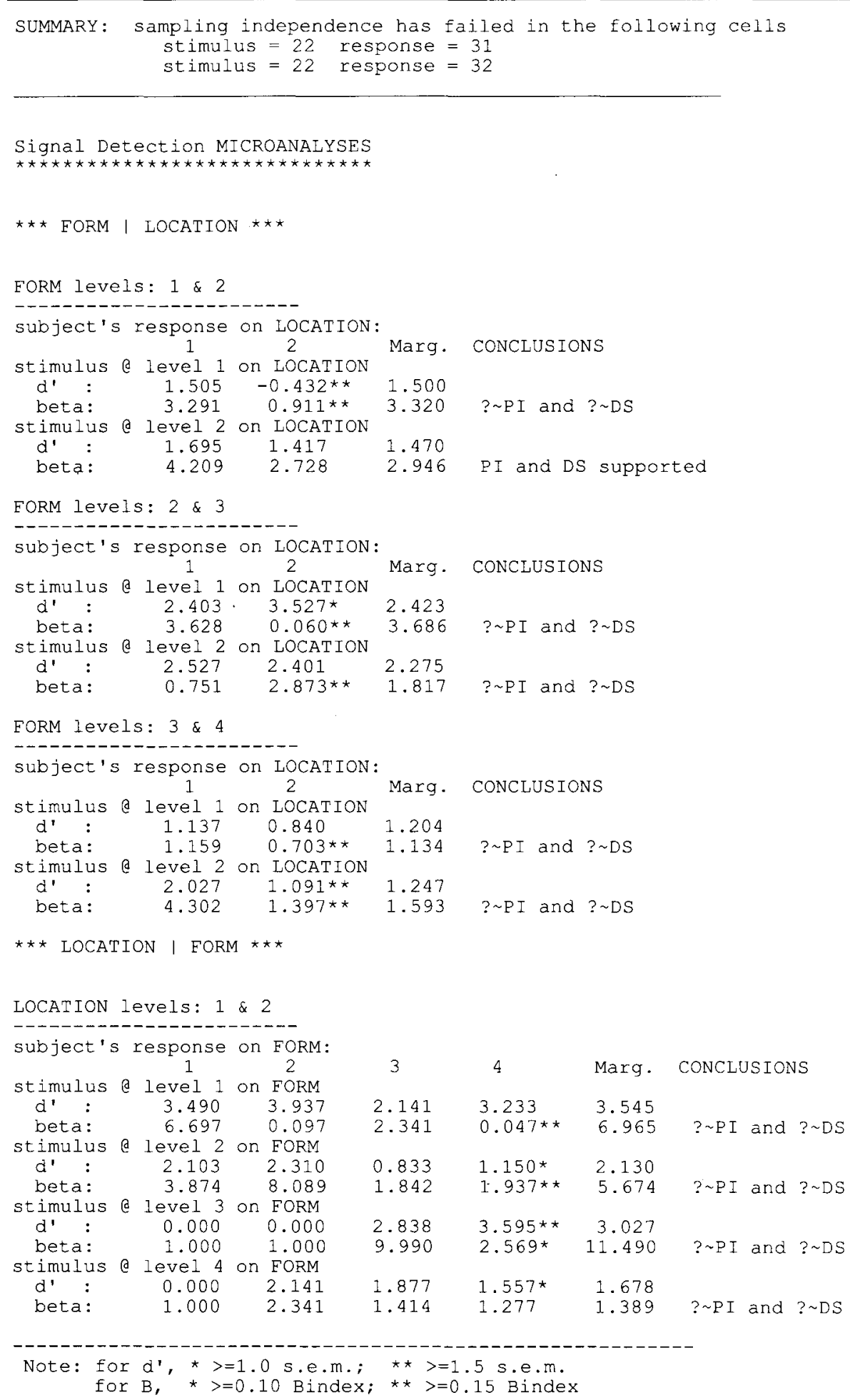

(Manuscript received May 25, 1994 